University of Nebraska - Lincoln

DigitalCommons@University of Nebraska - Lincoln

$1-1-2008$

\title{
The kinetic energy dependent effective Debye temperature for $\mathrm{CoS}_{2}(100)$
}

\author{
Ning Wu \\ University of Nebraska-Lincoln \\ David Wisbey \\ University of Nebraska-Lincoln \\ Takashi Komesu \\ University of Nebraska-Lincoln, tkomesu2@unl.edu \\ Z. X. Yu \\ Zhongshan University \\ M. Manno \\ University of Minnesota, Minneapolis, MN \\ See next page for additional authors
}

Follow this and additional works at: https://digitalcommons.unl.edu/physicsdowben

Part of the Physics Commons

Wu, Ning; Wisbey, David; Komesu, Takashi; Yu, Z. X.; Manno, M.; Wang, L.; Leighton, C.; and Dowben, Peter A., "The kinetic energy dependent effective Debye temperature for $\mathrm{CoS}_{2}$ (100)" (2008). Peter Dowben Publications. 198.

https://digitalcommons.unl.edu/physicsdowben/198

This Article is brought to you for free and open access by the Research Papers in Physics and Astronomy at DigitalCommons@University of Nebraska - Lincoln. It has been accepted for inclusion in Peter Dowben Publications by an authorized administrator of DigitalCommons@University of Nebraska - Lincoln. 


\section{Authors}

Ning Wu, David Wisbey, Takashi Komesu, Z. X. Yu, M. Manno, L. Wang, C. Leighton, and Peter A. Dowben 


\title{
The kinetic energy dependent effective Debye temperature for $\mathrm{CoS}_{2}(100)$
}

\author{
Ning $\mathrm{Wu}^{1}$, David Wisbey ${ }^{1}$, Takashi Komesu ${ }^{1}$, Z. X. Yu ${ }^{2}$, M. Manno ${ }^{3}$, \\ L. Wang ${ }^{3}$, C. Leighton ${ }^{3}$, and P. A. Dowben ${ }^{1, *}$ \\ ${ }^{1}$ Department of Physics and Astronomy and the Nebraska Center for Materials and Nanoscience, \\ University of Nebraska-Lincoln, Lincoln, NE 68588-0111, USA \\ ${ }^{2}$ School of Physics \& Engineering, Zhongshan University, Guangzhou 510275, People's Republic of China \\ ${ }^{3}$ Department of Chemical Engineering and Materials Science, University of Minnesota, Minneapolis, MN 55455, USA \\ * Corresponding author
}

\begin{abstract}
The effective Debye temperatures of the highly spin-polarized material $\mathrm{CoS}_{2}$ were measured using temperature dependent low energy electron diffraction and shown to be dependent upon electron kinetic energy. The normal dynamic motion of the (100) surface results in the effective surface Debye temperature of $326 \pm 9 \mathrm{~K}$ compared to a bulk Debye temperature of $612 \pm 24 \mathrm{~K}$. Similar values for the bulk Debye temperature have been obtained through LEED I(V) analysis, core level photoemission with a lower value for the bulk Debye temperature found from heat capacity measurements.
\end{abstract}

Keywords: Surface Debye temperature, Cobalt pyrite, Lattice vibrations

PACS classification codes: 68.35.Ja; 61.14.Ax; 71.20.Be; 79.60.-i

Small lattice distortions could have a profound influence on the spin asymmetry at the Fermi energy in highly polarized materials, as has been suggested for both $\mathrm{CoS}_{2}[1]$ and $\mathrm{NiMnSb}$ [2]. For $\mathrm{CoS}_{2}$, small displacements of the sulfur atom in the unit cell affect the position of the spin minority bands [1]. The placement of these bands with strong sulfur weight, with respect the Fermi level, can dramatically alter the polarization [1]. Furthermore, since the vibrational structure at both surfaces and interfaces can have a profound effect on the polarization [3] and polarization injection [4], low Debye temperatures, particularly at interfaces, are generally undesirable for spin filters and spin injection. Cobalt disulfide $\left(\mathrm{CoS}_{2}\right)$ has been measured to be highly spin polarized [5] with a Curie temperature in the range of 116-120 K [6], but the role of dynamic motion on the band structure and polarization has not yet been clearly established.

The Debye temperature is a key descriptive parameter of the dynamic motions of atoms on the surface, as well as in the bulk. The surface Debye temperature of single crystal materials can be investigated by low energy electron diffraction (LEED) [7-12], X-ray photoemission spectroscopy (XPS) or valence band photoemission (PES) [11-16], inverse photoemission [17] as well as atom beam scattering and other surface sensitive techniques. With LEED and the various electron spectroscopies, the electron mean free path plays a key role in establishing the effective Debye temperature [11] and [12], representative of motion along the surface normal. Generally, in electron scattering and electron spectroscopy techniques it is assumed, in the absence of surface phase transitions, that the emerging electron beam intensity depends exponentially on the sample temperature [7-17]

$$
I=I_{0} \exp (-2 \mathrm{~W})
$$

where $W$ is Debye-Waller factor given by

$$
2 W=|\Delta k|^{2}\left\langle u_{0}\right\rangle^{2}
$$


where $\Delta k$ is the wave vector transfer and $\left\langle u_{0}\right\rangle^{2}$ is the mean square displacement of the atoms. In the Debye model of thermal vibration, in the case of isotropic vibration, $W$ is described

$$
2 W=\frac{3 T(\hbar \Delta k)^{2}}{m k_{B} \theta_{D}^{2}}
$$

where $T$ is the sample temperature, $h(\Delta k)$ is the electron momentum transfer, $m$ is the mass of the scattering center, and $\theta_{D}$ is the effective Debye temperature. This Debye temperature is dominated by dynamic motions normal to the surface in almost all experiments and typically does not contain significant in plane or anharmonic contributions to the true Debye temperature.

In this "Letter," we investigate the Debye temperature of $\mathrm{CoS}_{2}(100)$ by LEED as a function of electron kinetic energy, comparing the data to temperature dependent XPS, and LEED $\mathrm{I}(\mathrm{V})$ analysis. Because of the different scattering geometries, we estimate the electron momentum transfer differently for LEED and XPS. For LEED, the momentum transfer is

$$
|\Delta \vec{k}|=\left|\vec{k}_{f}-\vec{k}_{i}\right|=\left|2 k \cos \frac{\theta}{2}\right|
$$

where $\theta$ is the angle between surface normal and diffracted electron beam [9], [10] and [15]. For the case of $\mathrm{CoS}_{2}$, as with other multicomponent crystals [7], [11] and [12], the mass of the scattering center in LEED is the average mass calculated from one cobalt atom and two sulfur atoms. Due to geometry of our LEED experiments here, the scattering vector is close to the surface normal, so $\theta$ is very small. For XPS, the momentum transfer is the momentum of the emitted photoelectron [11-16]. Since the photoelectrons were collected normal to the surface $(\theta=0)$, the vibrational motions normal to the surface are again the dominant contribution to the Debye-Waller factor. In XPS, the element of origin for emitted photoelectron determines the mass of the scattering centers [11], [12] and [15]. In our experiment, we monitored the photoelectron intensity from the $2 \mathrm{p}_{2 / 3}$ shell of the cobalt atoms and the $2 \mathrm{p}$ shell of the sulfur atoms (using $\mathrm{MgK}_{\alpha}$ radiation at $1253.6 \mathrm{eV}$ ). The X-ray photoelectron spectroscopy (XPS) studies were performed with a Gammadata Scienta SES-100 electron energy hemispherical analyzer and a SPECS X-ray source. LEED intensity versus voltage data, when complemented by dynamical scattering calculations (i.e. dynamical scattering analysis of the $\mathrm{I}(\mathrm{V})$ curves for multiple diffraction beams) can also be used to obtain a layer by layer estimate of the effective Debye temperature [7]. Such LEED I(V) analysis has already been used to determine the structure of the $\mathrm{CoS}_{2}(100)-(1 \times 1)$ surface [18] using an automated tensor LEED program [19] and [20].

The success of this work depends on a surface stoichiometry that is well characterized and not susceptible to surface segregation under experimental conditions. The surface of $\mathrm{CoS}_{2}$ has been established, with the surface stoichiometry preserved under our experimental conditions [18]. The preparation of the surface structure, schematically shown in the inset to Figure 2, was made possible by the cleavage of sufficiently large $\mathrm{CoS}_{2}$ (100) single crystals ( $\mathrm{mm}$ in diameter), prepared by chemical vapor transport, as detailed elsewhere [5]. These crystals, when cleaved, provide low energy electron diffraction (LEED) patterns characteristic of the highly ordered $1 \times 1$ surface [18].

Figure 1 shows the temperature dependent LEED intensities, after background $\left(I_{\mathrm{bg}}\right)$ subtraction and normalization to the value $I_{0}$ at the lowest temperature, for two different electron energies $(107 \mathrm{eV}$ and $121 \mathrm{eV})$. Multiple sets of data were taken at six different incident electron energies $(89,107,121$, 167, 213 and $222 \mathrm{eV}$ ). The effective Debye temperatures were derived from the data to be $326 \pm 9 \mathrm{~K}$ (at $89 \mathrm{eV}$ incident electron energy), $405 \pm 8 \mathrm{~K}$ (at $107 \mathrm{eV}$ ), $460 \pm 7 \mathrm{~K}$ (at $121 \mathrm{eV}$ ), $542 \pm 21 \mathrm{~K}$ (at $167 \mathrm{eV}), 595 \pm 23 \mathrm{~K}$ (at $213 \mathrm{eV}$ ) and $612 \pm 24$ $\mathrm{K}$ (at $222 \mathrm{eV}$ ), using Eq. (1). These six different Debye temperatures have been plotted against electron energies in Figure 2 (panel (a)). As the incident electron energy increases so does the electron mean free path and effective probing depth [10] and [21]. Thus a smaller electron kinetic energy should be more surface sensitive, and larger electron kinetic energy would be more dominated by the bulk.

The inelastic mean free path $\lambda$ can be roughly described as [21], [22] and [23]:

$$
\lambda=\frac{E}{E_{p}^{2}\left[\beta \ln (\gamma E)-(C / E)+\left(D / E^{2}\right)\right]}
$$

where $E$ is electron kinetic energy, $E_{p}=28.8\left(N_{\nu} \rho / M\right)^{1 / 2}$ is roughly the free electron plasmon energy, $N_{v}$ is the number of valence electrons per atom, $M$ is the atomic or molecular weight, $\rho$ is the density and $\beta, \gamma, C$ and $D$ are fitting parameters that can be expressed as:

$$
\begin{aligned}
& \beta=-0.1+0.944 /\left(E_{p}^{2}+E_{g}^{2}\right)^{1 / 2}+0.069 \rho^{0.1}, \\
& \gamma=0.191 \rho^{-0.5} \\
& C=1.97-0.91 U, \\
& D=53.4-20.8 U, \\
& U=N_{v} \rho / M=E_{p}^{2} / 829.4
\end{aligned}
$$

where $E_{g}$ is the bandgap energy. This ensemble of equations (sometime known as TPP-2M) [22] and [23], may be used to assess the mean free path of in the surface region of $\mathrm{CoS}_{2}$, exploiting NIST Electron Inelastic Mean Free Path database (version 1.1) [24]. The effective attenuation length (EAL) has been calculated using the approach of Seah [25]:

$$
\mathrm{EAL}=\lambda_{i}\left(1-0.028 Z^{0.5}\right)[0.501+0.068 \ln (E)]
$$

where $\lambda_{i}$ is inelastic mean free path, $Z$ is atomic number of the compound and $E$ is electron kinetic energy. We have to divide this effective attenuation length by factor 2 to because the collected electrons from LEED experiments go through the surface region twice (in and out), and is partly the basis for the much greater surface sensitivity of LEED than XPS for a given energy. The change of inelastic mean free path (IMFP) and effective attenuation length (EAL), for $\mathrm{CoS}_{2}(100)$, as a function of the kinetic energy of incident electrons are summarized in Figure 2(b) for several choices of the number of valence electrons per chemical formulae unit $(7,4$, and 1$)$.

Caution should be used in interpreting these numbers, not simply because this is an approximation but also because this 

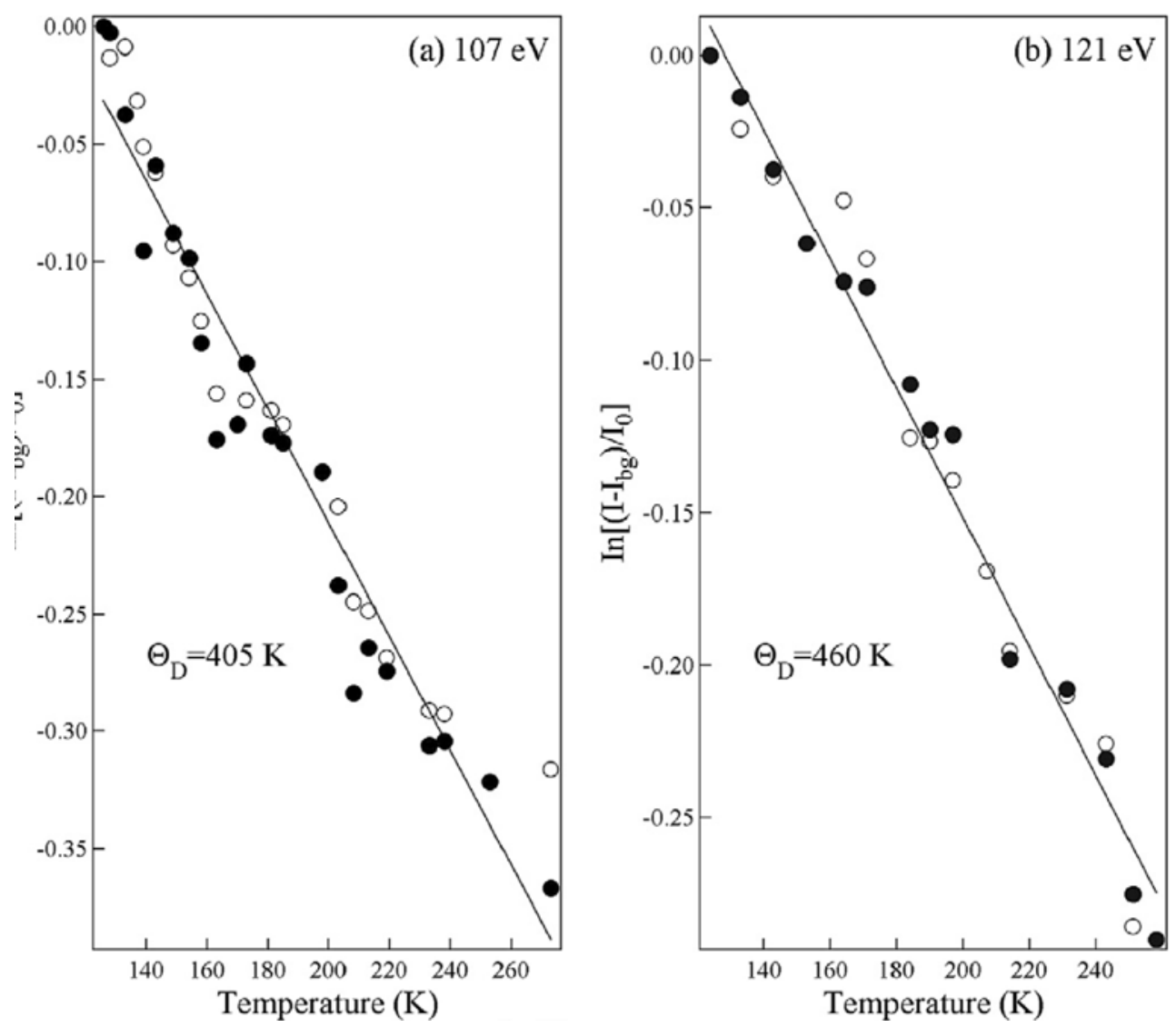

Figure 1. Logarithm of the intensities of the diffraction elections spots obtained in LEED as a function of temperature, after background $\left(I_{\mathrm{bg}}\right)$ subtraction and normalization to the value $\left(I_{0}\right)$ at the lowest temperature. Two different incident electron energies are shown in (a) $107 \mathrm{eV}$ and (b) $121 \mathrm{eV}$, with two representative sets of data (open and solid circles) for each energy. The experiment values are fitted (solid line) with the Debye-Waller factor using Eqs. (1) and (3), as described in the text.

equation describes effective attenuation length from overlayer-film samples that are measured by photoemission techniques. Admittedly, this is an imperfect model for calculating effective attenuation lengths as discrepancies are very evident between the slope of fitting curve in Figure 2(a) and that of probing depth in Figure 2(b). This comparison between experiment and theory indicates that a calculation of attenuation length based on the valence electron count is fraught with uncertainty and that a better method for calculation attenuation lengths needs to be derived, particularly at lower electron kinetic energies. The modeling methodologies need to be better than just a plasmon loss model based on electron count for compound systems. Compound systems like $\mathrm{Fe}_{3} \mathrm{O}_{4}$ (also a high polarization ferromagnet) can exhibit a dramatic logarithmic dependence on electron energy than other metals [26]. What exactly is the electron count contributing to inelastic electron losses due to plasmons and what are the matrix elements for the other various loss mechanisms is not clear and not well defined [27]. We have been able to show already that core can play a role in the plasmon structure for poor metals [28]. Experiment must be the ruler.

As the effective attenuation length is generally experimentally seen to be a logarithmic function of the electron kinetic energy [25] and [26], we have fitted the extracted experimental effective Debye temperature, as a function of electron ki- netic energy, to a logarithmic function, as shown in Figure 2(a). Therefore there should be a layer dependence of the Debye temperature, and under no conditions for the work here is LEED perfectly sensitive to the surface or bulk alone. Nonetheless, the general trend is clear from Figure 2, and there is general agreement with our other measurements of the Debye temperature.

Our effective surface Debye temperature of $326 \pm 9 \mathrm{~K}$ obtained at $89 \mathrm{eV}$ incident electron energy, which should be more representative of the surface, is reasonably consistent with the surface Debye temperature values of $350 \mathrm{~K}$ and $460 \mathrm{~K}$ for cobalt and sulfur respectively, obtained from the LEED I(V) analysis for data taken at room temperature alone, as partly described elsewhere [18]. These estimates for the Debye temperatures, obtained from the LEED I(V) analysis, consider multiple scattering, and we note that there are associated complications may affect the value and accuracy of the Debye temperature estimated in this fashion. The Debye temperature extracted from the LEED I(V) analysis employs the experimental data taken at room temperature and the effective Debye temperature is just an adjust parameter in the LEED I(V) analysis and obtained from a optimization procedure. In the kinematic limit, the Debye-Waller formalism applies and the Debye temperature extracted in this manner depends on the temperature itself. In the case of surfaces, the Debye temperature will also depend on anisotropic vibrational contributions and also have 

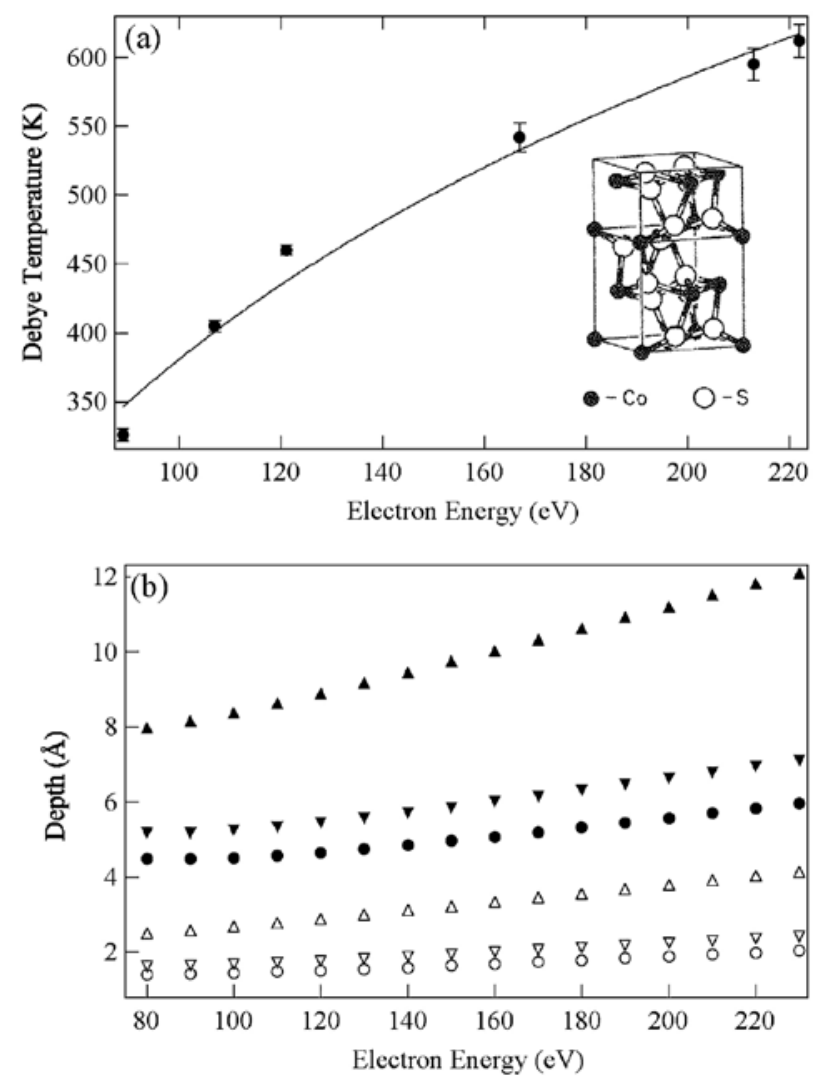

Figure 2. Panel (a): Debye temperatures extracted from LEED as a function of incident electron energies. Schematic diagram of the sulfur terminated surface is shown in the inset, adapted from [36]. Panel (b): the inelastic mean free path (filled symbols) and effective attenuation length (open symbols) plotted as a function of incident electron energies, for seven (circles: $\lambda \mu$ ), four (down triangles: $\tau \nabla$ ) and one (up triangles: $\sigma \Delta$ ) [per chemical formulae].

some layer dependence. Regrettably, the thermal lattice expansion due to anharmonic vibrational effects difficult to assess in low energy electron diffraction and LEED I(V) analysis.

Figure 3 shows the logarithm of the core level intensities as a function of temperature for Co $2 p_{3 / 2}$ and $S 2 p$ core levels obtained by X-ray photoemission. The electron kinetic energies for XPS are much higher and should be more representative of the bulk as the Co $2 \mathrm{p}_{3 / 2}$ and Co $2 \mathrm{p}_{1 / 2}$ core levels (shown as insets in Figure 3) are located at binding energies of 778.3 $\pm 0.2 \mathrm{eV}$ and $793.4 \pm 0.2 \mathrm{eV}$ respectively, while the $\mathrm{S} 2 \mathrm{p}_{3 / 2}$ is at a binding energy $162.6 \pm 0.2 \mathrm{eV}$. The effective bulk Debye temperatures are $555 \pm 21 \mathrm{~K}$, derived from Co $2 \mathrm{p}_{3 / 2}$ core level intensity, and $511 \pm 26 \mathrm{~K}$, derived from S $2 \mathrm{p}$ core levels. The bulk Debye temperature values obtained from XPS are slightly smaller than the $600 \mathrm{~K}$ for cobalt and $800 \mathrm{~K}$ for sulfur derived from LEED I(V) analysis and the value of $612 \pm 24$ $\mathrm{K}$ obtained at $222 \mathrm{eV}$ electron kinetic energy from temperature dependent LEED. In general, the Debye temperatures obtained from XPS for sulfur are higher than those obtained for cobalt, in part reflecting the higher electron kinetic energies (i.e. outgoing electron energies of roughly $464 \mathrm{eV}$ for cobalt versus $1092 \mathrm{eV}$ for sulfur).

These values for the bulk Debye temperatures, using LEED and electron spectroscopy are significantly higher than the val-
Table 1. $\mathrm{CoS}_{2}$ (100) Debye temperatures

\begin{tabular}{lcl}
\hline Method and analysis technique & $\begin{array}{l}\text { Surface Debye } \\
\text { temperature }\end{array}$ & $\begin{array}{l}\text { Bulk Debye } \\
\text { temperature }\end{array}$ \\
\hline $\begin{array}{l}\text { LEED scattering intensities versus } \\
\text { temperature }\end{array}$ & $326 \pm 9 \mathrm{~K}$ & $612 \pm 24 \mathrm{~K}$ \\
$\begin{array}{l}\text { Tensor LEED I(V) analysis: cobalt } \\
\text { Tensor LEED I(V) analysis: sulfur }\end{array}$ & $350 \mathrm{~K}$ & $600 \mathrm{~K}$ \\
$\begin{array}{l}\text { XPS intensities versus temperature: } \\
\text { cobalt }\end{array}$ & $460 \mathrm{~K}$ & $800 \mathrm{~K}$ \\
$\begin{array}{l}\text { XPS intensities versus temperature: } \\
\text { sulfur }\end{array}$ & & $555 \pm 21 \mathrm{~K}$ \\
Bulk heat capacity & & $511 \pm 26 \mathrm{~K}$ \\
\hline
\end{tabular}

ues for the bulk Debye temperature of $489 \pm 5.1 \mathrm{~K}$, obtained from heat capacity measurements (as has been done for polycrystalline samples [6]) taken from $\mathrm{CoS}_{2}$ single crystals in the temperature range 1.8 to $200 \mathrm{~K}$. These latter values of the Debye temperature should be less than those obtained from LEED and XPS, as the measurement should include contributions along other crystallographic directions and significant anharmonic motion. This is one outlier in the bulk Debye temperature but the heat capacity measurement is measuring an overall Debye temperature, is not just measurement characteristic of normal motion along (100). This comparison of Debye temperature values is summarized in Table 1.

When comparing the Debye temperatures for the surface and the bulk, we may use a simple assumption that the surface vibration amplitudes $\left(\left\langle u_{0}\right\rangle^{2}\right.$ in Eq. (2)) is enhanced when the number of nearest atoms surrounding surface atoms is halved compared with neighbor atoms of bulk atoms [10]. The negative correlation between vibration amplitudes and Debye temperature indicates that the Debye temperature for bulk is $\sqrt{2}$ times greater than that for surface. Considering the uncertainties in the absolute values and accuracy of the derived Debye temperature, these Debye temperatures for the surface and the bulk differ by far more than the expected simple geometrical factor of $\sqrt{2}$ [10]. For a close packed surface, these general arguments imply that the Debye temperatures for the surface and the bulk differ by less than a simple geometrical factor of $\sqrt{2}$.

Effective surface and bulk Debye temperature differ dramatically when the composition of the surface is vastly different from the bulk. This might explain the prior results for $\mathrm{La}_{0.35} \mathrm{~Pb}_{0.65} \mathrm{MnO}_{3}$ (100) [12], but such an explanation cannot be applied here or in the case of ErAs (100) films [11], as the stoichiometry of the surface is that of the bulk. What these result tends to indicate is that there is a surface layer relaxation for ErAs (100) [11] and $\mathrm{CoS}_{2}$ (100), which although small [18], permits the surface atoms to exhibit large amplitude soft vibrational modes of low energy along the surface normal.

Because of plasmon-magnon and magnon-phonon interactions, the spin injections will also clearly be dependent upon electron energy, particularly in the hot electron regime, without even the additional considerations necessary due to the details of the electronic structure at the interface of $\mathrm{CoS}_{2}$ and a semiconductor. Strong inelastic scattering of the electron from particle-hole and collective excitations (plasmons) will in short mean free paths that can be spin dependent [29-33]. 

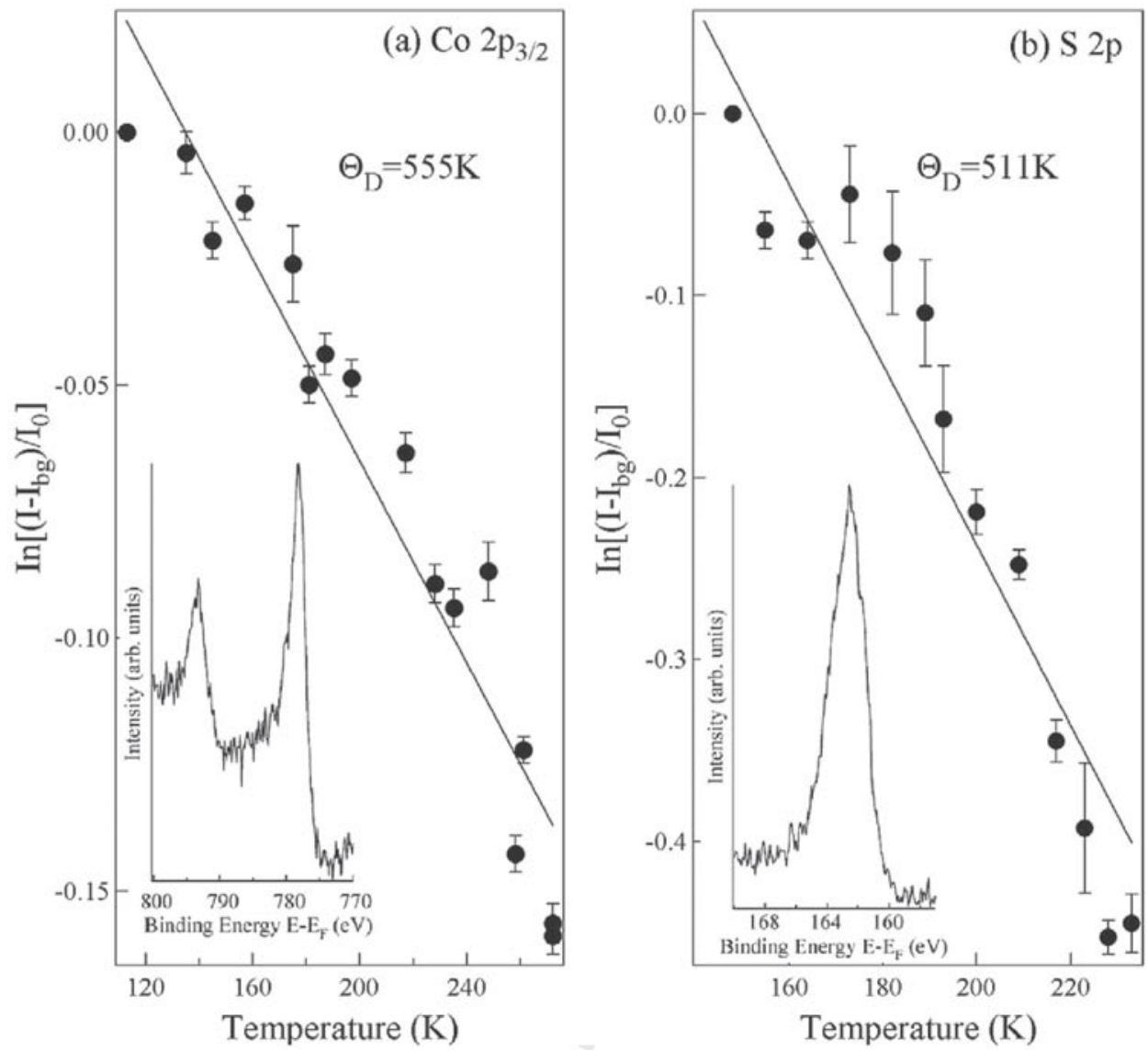

Figure 3. Logarithm of the Co $2 \mathrm{p}_{3 / 2}$ (a) and S $2 \mathrm{p}$ (b) core level photoemission intensities as a function of temperature, after background ( $\left.I_{\mathrm{bg}}\right)$ subtraction and normalization to the value $\left(I_{0}\right)$ at the lowest temperature. The experiment values are fitted (solid line) with the Debye-Waller factor using Eqs. (1) and (3). The insets show X-ray photoemission spectra of Co $2 \mathrm{p}_{3 / 2}$ (a) and S $2 \mathrm{p}$ (b) core level.

Furthermore, understanding of the spin dependence of the inelastic mean free path is critical to the interpretation of results from spin-polarized electron spectroscopies since plasmonmagnon coupling can occur [34]. In high polarization materials, magnon-phonon coupling can also occur [35], and is seen in materials with Debye temperatures little different (where known) from $\mathrm{CoS}_{2}$, as reported here. Particularly pertinent to the discussion here, we note that in high polarization materials there is a delicate balance of energies to maintain the high values of electron polarization at the Fermi level, as small adjustments in atomic positions may have profound effects upon the density of states in the minority spin channel. Such lattice distortions can occur with anharmonic vibrational motion of the lattice so that the low Debye temperature may implicate a phonon mediated reduction to spin injection and spin polarization in this and related materials. Clearly a simple plasmon model for estimating the electron effective attenuation length is insufficient, as is demonstrated here.

In conclusion, we have found the effective Debye temperature for $\mathrm{CoS}_{2}(100)$ single crystals varies roughly as the logarithm of the incident electron kinetic energy, or proportional to the expected functional for the elastic mean free path. The experimental values obtained from temperature dependent LEED are in general agreement with expectations from LEED $\mathrm{I}(\mathrm{V})$ analysis, from data taken from room temperature alone, temperature dependent XPS and heat capacity. While there care few experimental determinations of the surface Debye temperature for compound systems, usually because of problems with surface characterization and preparation, we have also been able to estimate a Debye temperature descriptive of motion largely along the surface normal for $\mathrm{CoS}_{2}(100)$.

\section{Acknowledgements}

This research was supported through the ONR Grant No. N00014-06-1-0616, the Nebraska Research Initiative and the NSF "QSPINS" MRSEC (DMR 0213808) at UNL, the work at UMN was supported by the NSF MRSEC under DMR0212302. The authors acknowledge the assistance of M.A. van Hove and Danqin Feng and helpful discussions with Kirill Beleschenko, and Wei-Ning Mei.

\section{References}

[1] N. Wu, Ya.B. Losovyj, D. Wisbey, K. Belashchenko, M. Manno, L. Wang, C. Leighton and P.A. Dowben, J. Phys.: Condens. Matter 19 (2007), p. 156224.

[2] M. Lezaic, P. Mavropoulos, J. Enkovaara, G. Bihlmayer and S. Blugel, Phys. Rev. Lett. 97 (2006), p. 026404.

[3] P.A. Dowben and R. Skomski, J. Appl. Phys. 95 (2004), p. 7453. 
[4] H. Itoh, T. Ohsawa and J. Inoue, Phys. Rev. Lett. 84 (2000), p. 2501.

[5] L. Wang, T.Y. Chen, C.L. Chien and C. Leighton, Appl. Phys. Lett. 88 (2006), p. 232509.

[6] L. Wang, T.Y. Chen, C.L. Chien, J.G. Checkelsky, J.C. Eckert, E.D. Dahlberg, K. Umemoto, R.M. Wentzcovitch and C. Leighton, Phys. Rev. B 73 (2006), p. 144402.

[7] E.A. Soares, V.E. de Carvalho and V.B. Nascimento, Surf. Sci. 431 (1999), p. 74.

[8] S. Mroz and A. Mroz, Surf. Sci. 109 (1981), p. 444.

[9] M.A. Van Hove, W.H. Weinberg and C.-M. Chan, Low Energy Electron Diffraction, Springer Series in Surface Science vol. 6, New York (1986), p. 134.

[10] L.J. Clarke, Surface Crystallography, Wiley, New York (1985).

[11] H.-K. Jeong, T. Komesu, P.A. Dowben, B.D. Schulz and C.J. Palmstrøm, Phys. Lett. A 302 (2002), p. 217.

[12] C.N. Borca, B. Xu, T. Komesu, H.-K. Jeong, M.T. Liu, S.H. Liou and P.A. Dowben, Surf. Sci. 512 (2002), p. L346.

[13] N.J. Shevchik, Phys. Rev. B 16 (1977), p. 3428.

[14] C. Waldfried, D.N. McIlroy, J. Zhang, P.A. Dowben, G.A. Katrich and E.W. Plummer, Surf. Sci. 363 (1996), p. 296.

[15] C.N. Borca, D. Ristoiu, T. Komesu, H.-K. Jeong, Ch. Hordequin, J. Pierre, J.P. Nozieres and P.A. Dowben, Appl. Phys. Lett. 77 (2000), p. 88.

[16] R.S. Williams, P.S. Wehner, J. Stohr and D.A. Shirley, Phys. Rev. Lett. 39 (1977), p. 302.

[17] J. Choi, P.A. Dowben, S. Pebley, A.V. Bune, S. Ducharme, V.M. Fridkin, S.P. Palto and N. Petukhova, Phys. Rev. Lett. 80 (1998), p. 1328.

[18] Z.X. Yu, M.A. Van Hove, S.Y. Tong, D. Wisbey, Ya.B. Losovyj, N. Wu, M. Manno, L. Wang, C. Leighton, W.N. Mei and P.A. Dowben, J. Phys.: Condens. Matter 19 (2007), p. 156223.

[19] D.K. Saldin and P.L. de Andres, Phys. Rev. Lett. 64 (1990), p. 1270.

[20] P.J. Rous, J.B. Pendry, D.K. Saldin, K. Heinz, K. Muller and N. Bickel, Phys. Rev. Lett. 57 (1986), p. 2951.
[21] A. Jablonski and C.J. Powell, Surf. Sci. Rep. 47 (2002), p. 33.

[22] S. Tanuma, C.J. Powell and D.R. Penn, Surf. Interface Anal. 21 (1994), p. 165.

[23] C.J. Powell and A. Jablonski, Surf. Interface Anal. 29 (2000), p. 108.

[24] NIST Electron Inelastic Mean Free Path program version 1.1 (NST std. ref. Database 71), http://www.nist.gov.srd.

[25] M.P. Seah, Practical Surface Analysis, vol. 1, Auger and X-ray Photoelectron Spectroscopy, Wiley, Chichester, UK (1990) p. 201

[26] C.J. Powell, Surf. Sci. 44 (1974), p. 29.

[27] C.N. Borca, R.H. Cheng, S. Stadler, Y.U. Idzerda, J. Choi, D.N. McIlroy, Q.L. Xu, S.H. Liou, Z.C. Zhong and P.A. Dowben In: M. Rzchowski, M. Kawasaki, A.J. Millis, M. Rajeswari and S. von Molnár, Editors, Magnetoresistive Oxides and Related Materials, MRS Symposium Proceedings vol. 602 (2001), pp. 301306 and references therein.

[28] B.-O. Kim, G. Lee, E.W. Plummer, P.A. Dowben and A. Liebsch, Phys. Rev. B 52 (1995), p. 6057.

[29] D.P. Pappas, K.-P. Kämper, B.P. Miller, H. Hopster, D.E. Fowler, C.R. Brundle, A.C. Luntz and Z.-X. Shen, Phys. Rev. Lett. 66 (1991), p. 504.

[30] E. Vescovo, C. Carbone, U. Alkemper, O. Rader, T. Kachel, W. Gudat and W. Eberhardt, Phys. Rev. B 52 (1995), p. 13497.

[31] H. Hopster, R. Raue, E. Kisker, G. Güntherodt and M. Campagna, Phys. Rev. Lett. 50 (1983), p. 70.

[32] M.P. Gokhale and D.L. Mills, Phys. Rev. Lett. 66 (1991), p. 2251.

[33] J. Hong and D.L. Mills, Phys. Rev. B 59 (1999), p. 13840.

[34] R. Skomski and P.A. Dowben, J. Appl. Phys. 99 (2006), p. $08 \mathrm{~F} 508$.

[35] P.A. Dowben and S.J. Jenkins In: A. Narlikar, Editor, Frontiers in Magnetic Materials, Springer-Verlag (2005).

[36] Y.J. Jin and J.I. Lee, Phys. Rev. B 73 (2006), p. 064405. 